\title{
Sur la capitulation des 2-classes d'idéaux de $\mathbb{k}=\mathbb{Q}(\sqrt{2 p q}, i)$ où $p \equiv-q \equiv 1 \bmod 4$ \\ par
}

\author{
Abdelmalek Azizi (Oujda)
}

1. Introduction. Soient $\mathbb{k}$ un corps de nombres de degré fini sur $\mathbb{Q}, \mathbb{F}$ une extension non ramifiée de $\mathbb{k}$ et $p$ un nombre premier. L'extension $\mathbb{k}^{(1)}$ de $\mathbb{k}$, abélienne maximale et non ramifiée pour tous les idéaux premiers finis et infinis, est dite corps de classes de Hilbert de $\mathbb{k}$. De même l'extension $\mathbb{k}_{p}^{(1)}$ de $\mathbb{k}$ dont le degré est une puissance de $p$, abélienne maximale et non ramifiée pour tous les idéaux premiers finis et infinis est dite $p$-corps de classes de Hilbert de $\mathbb{k}$.

La recherche des idéaux de $\mathbb{k}$ qui capitulent dans $\mathbb{F}$ (deviennent principaux dans $\mathbb{F}$ ) a été l'objet d'étude d'un grand nombre de mathématiciens. En effet, Kronecker était parmi les premiers à avoir abordé des problèmes de capitulation dans le cas des corps quadratiques imaginaires. Dans le cas où $\mathbb{F}$ est égal au corps de classes de Hilbert $\mathbb{k}^{(1)}$ de $\mathbb{k}$, D. Hilbert avait conjecturé que toutes les classes de $\mathbb{k}$ capitulent dans $\mathbb{k}^{(1)}$ (théorème de l'idéal principal). La preuve de ce dernier théorème a été réduite par E. Artin à un problème de la théorie des groupes, et c'est Ph. Furtwängler qui l'avait achevée.

Le cas où $\mathbb{F} / \mathbb{k}$ est une extension cyclique de degré un nombre premier $p$ a été traité par Hilbert. Sa réponse est le sujet du théorème 94 qui affirme qu'il y a au moins une classe non triviale dans $\mathbb{k}$ qui capitule dans $\mathbb{F}$. De plus, Hilbert avait trouvé le résultat suivant :

Soient $\sigma$ un générateur du groupe de Galois de $\mathbb{F} / \mathbb{k}, N$ la norme de $\mathbb{F} / \mathbb{k}, \mathbf{U}_{0}$ le groupe des unités de $\mathbb{k}$, $\mathbf{U}$ le groupe des unités de $\mathbb{F}$ et $\mathbf{U}^{*}$ le sous-groupe des unités de $\mathbf{U}$ dont la norme, relative à l'extension $\mathbb{F} / \mathbb{k}$, est égale à 1 . Alors le groupe des classes de $\mathbb{k}$ qui capitulent dans $\mathbb{F}$ est isomorphe au groupe quotient $\mathbf{U}^{*} / \mathbf{U}^{1-\sigma}=\mathbf{H}^{1}(\mathbf{U})$, le groupe cohomologique de $\mathbf{U}$ de dimension 1.

2000 Mathematics Subject Classification: 11R27, 11R37.

Key words and phrases: groupe des unités, système fondamental d'unités, capitulation, corps de classes de Hilbert. 
A l'aide de ce théorème et de plusieurs résultats sur les groupes cohomologiques des unités, on montre le théorème suivant :

ThÉORÈme 1 . Soit $\mathbb{F} / \mathbb{k}$ une extension cyclique de degré un nombre premier, alors le nombre des classes qui capitulent dans $\mathbb{F} / \mathbb{k}$ est égal à $[\mathbb{F}: \mathbb{k}]\left[\mathbf{U}_{0}: N(\mathbf{U})\right]$.

Soient $\mathbb{k}$ un corps de nombres, $\mathbf{C}_{\mathbb{k}}$ le groupe de classes de $\mathbb{k}, \mathbf{C}_{2}$ la 2composante du groupe de classes de $\mathbb{k}, \mathbb{k}_{2}^{(1)}$ le 2-corps de classes de Hilbert de $\mathbb{k}, \mathbb{k}_{2}^{(2)}$ le 2-corps de classes de Hilbert de $\mathbb{k}_{2}^{(1)}$ et $G_{2}$ le groupe de Galois de $\mathbb{k}_{2}^{(2)} / \mathbb{k}$.

Définition 2. Soient $\mathbb{M}$ une extension cyclique non ramifiée de $\mathbb{k}, \mathbf{C}_{\mathbb{M}}$ le groupe de classes de $\mathbb{M}$ et $\mathbf{C}_{\mathbb{k}, \mathbb{M}}$ le sous-groupe de $\mathbf{C}_{\mathbb{k}}$ associé à $\mathbb{M}$ par la théorie du corps de classes. On dit que $\mathbb{M}$ est

- de type $(A)$ si un élément de $\mathbf{C}_{\mathbb{k}, \mathbb{M}}$ capitule dans $\mathbb{M}$,

- de type $(B)$ si aucun élément de $\mathbf{C}_{\mathbb{k}, \mathbb{M}}$ ne capitule dans $\mathbb{M}$.

Soient $j$ l'application de $\mathbf{C}_{\mathbb{k}}$ vers $\mathbf{C}_{\mathbb{M}}$ qui fait correspondre à la classe d'un idéal $\mathcal{A}$ de $\mathbb{k}$ l'idéal engendré par $\mathcal{A}$ dans $\mathbb{M}$ et $N$ la norme de $\mathbb{M} / \mathbb{k}$; alors on a :

$$
\begin{aligned}
& \mathbb{M} \text { est de type }(\mathrm{A}) \Leftrightarrow\left|\operatorname{Ker}(j) \cap N\left(\mathbf{C}_{\mathbb{M}}\right)\right|>1, \\
& \mathbb{M} \text { est de type }(\mathrm{B}) \Leftrightarrow\left|\operatorname{Ker}(j) \cap N\left(\mathbf{C}_{\mathbb{M}}\right)\right|=1 .
\end{aligned}
$$

DÉfinition 3. Soient $Q_{m}$ le groupe des quaternions, $D_{m}$ le groupe diédral et $S_{m}$ le groupe semi-diédral d'ordre $2^{m}$. Ces groupes sont définis comme suit : chaque groupe est engendré par deux éléments $x$ et $y$ tels que :

$$
\begin{aligned}
Q_{m}=\langle x, y\rangle & \text { où } x^{2^{m-2}}=y^{2}=a, a^{2}=1, y^{-1} x y=x^{-1}, \\
D_{m}=\langle x, y\rangle & \text { où } x^{2^{m-1}}=y^{2}=1, y^{-1} x y=x^{-1}, \\
S_{m}=\langle x, y\rangle & \text { où } x^{2^{m-1}}=y^{2}=1, y^{-1} x y=x^{2^{m-2}-1} .
\end{aligned}
$$

On sait que si $G_{2}$ est d'ordre $2^{m}, m>1$ et $G_{2} / G_{2}^{\prime} \simeq \mathbb{Z} / 2 \mathbb{Z} \times \mathbb{Z} / 2 \mathbb{Z}$, alors $G_{2}$ est isomorphe à $Q_{m}, D_{m}$ ou $S_{m}$. Dans tous ces cas, on a $G_{2}^{\prime}=\left\langle x^{2}\right\rangle$ et les trois sous-groupes d'indice 2 de $G_{2}$ sont : $H_{1}=\langle x\rangle, H_{2}=\left\langle x^{2}, y\right\rangle$ et $H_{3}=\left\langle x^{2}, x y\right\rangle$. Soient $\mathbb{F}_{i}$ le sous-corps de $\mathbb{k}_{2}$ laissé fixe par $H_{i}$ et $j_{i}$ l'application $j$ définie pour $\mathbb{M}=\mathbb{F}_{i}$. Si $G_{2}^{\prime} \neq 1$, alors il existe un sous-groupe $\left\langle x^{4}\right\rangle$ de $G_{2}^{\prime}$ d'indice 2 engendré par $x^{4}$. Soit $\mathbb{L}$ le sous-corps de $\mathbb{k}_{2}$ laissé fixe $\operatorname{par}\left\langle x^{4}\right\rangle$.

ThÉorème 4 . On suppose que $G_{2} / G_{2}^{\prime} \simeq \mathbb{Z} / 2 \mathbb{Z} \times \mathbb{Z} / 2 \mathbb{Z}$. Alors on $a$ :

(i) Si $\mathbb{k}_{2}=\mathbb{k}_{1}$, alors les corps $\mathbb{F}_{i}$ sont de type $(A),\left|\operatorname{Ker}\left(j_{i}\right)\right|=4$ pour $i=1,2,3$ et $G_{2} \simeq \mathbb{Z} / 2 \mathbb{Z} \times \mathbb{Z} / 2 \mathbb{Z}$. 
(ii) Si $\operatorname{Gal}(\mathbb{L} / \mathbb{k}) \simeq Q_{3}$, alors $\mathbb{F}_{i}$ est de type $(A),\left|\operatorname{Ker}\left(j_{i}\right)\right|=2$ pour $i=1,2,3$ et $G_{2} \simeq Q_{3}$.

(iii) Si Gal $(\mathbb{L} / \mathbb{k}) \simeq D_{3}$, alors $\mathbb{F}_{2}$ et $\mathbb{F}_{3}$ sont de type $(B)$ et $\left|\operatorname{Ker}\left(j_{2}\right)\right|=$ $\left|\operatorname{Ker}\left(j_{3}\right)\right|=2$. De plus, si $\mathbb{F}_{1}$ est de type $(B)$ alors $\left|\operatorname{Ker}\left(j_{1}\right)\right|=2$ et $G_{2} \simeq S_{m}$. Si $\mathbb{F}_{1}$ est de type $(A)$ et $\left|\operatorname{Ker}\left(j_{1}\right)\right|=2$, alors $G_{2} \simeq Q_{m}$. Enfin si $\mathbb{F}_{1}$ est de type $(A)$ et $\left|\operatorname{Ker}\left(j_{1}\right)\right|=4$, alors $G_{2} \simeq D_{m}$.

On trouve plus d'informations sur ce dernier théorème dans [Ki-76]. On trouve d'autres résultats sur le problème de capitulation dans [Te-71], [Ki-76], [Mi-89], [Su-91] et [Az-97].

On désigne par $p$ un nombre premier congru à 1 modulo $4, q$ un nombre premier congru à -1 modulo $4, \mathbb{k}=\mathbb{Q}(\sqrt{2 p q}, i), \mathbb{k}_{2}^{(1)}$ le 2 -corps de classes de Hilbert de $\mathbb{k}, \mathbb{k}_{2}^{(2)}$ le 2-corps de classes de Hilbert de $\mathbb{k}_{2}^{(1)}$ et $G_{2}$ le groupe de Galois de $\mathbb{k}_{2}^{(2)} / \mathbb{k}$. D'après [Az-93] ou [Az-99:1], on a : $\mathbf{C}_{2} \simeq \mathbb{Z} / 2 \mathbb{Z} \times \mathbb{Z} / 2 \mathbb{Z}$ si et seulement si au moins deux éléments de $\left\{\left(\frac{2}{p}\right),\left(\frac{2}{q}\right),\left(\frac{p}{q}\right)\right\}$ valent -1 et l'indice $Q$ des unités de $\mathbb{Q}(\sqrt{2 p q})$ dans $\mathbb{k}$ est égal à 1 . Dans toute la suite on va donner un ensemble de résultats pour démontrer le théorème principal suivant :

ThÉORÈme PRINCIPAL. Soient $\mathbb{k}=\mathbb{Q}(\sqrt{2 p q}, i)$ et $\mathbf{C}_{2}$ la 2-partie $d u$ groupe de classes de $\mathbb{k}$. On suppose que $\mathbf{C}_{2} \simeq \mathbb{Z} / 2 \mathbb{Z} \times \mathbb{Z} / 2 \mathbb{Z} ;$ alors $\mathbb{k}_{2}^{(1)}$ contient trois extensions quadratiques de $\mathbb{k}, \mathbb{K}_{1}, \mathbb{K}_{2}$ et $\mathbb{K}_{3}$ où seulement deux classes de $\mathbf{C}_{2}$ capitulent et de plus $G_{2} \simeq Q_{m}$.

\section{Unités de certains corps de nombres de degré 8 sur $\mathbb{Q}$}

2.1. Préliminaires. Soient $d_{1}$ et $d_{2}$ deux entiers naturels sans facteurs carrés et premiers entre eux, $d_{3}=d_{1} d_{2}, \varepsilon_{1}$ (resp. $\varepsilon_{2}, \varepsilon_{3}$ ) l'unité fondamentale de $\mathbb{k}_{1}=\mathbb{Q}\left(\sqrt{d_{1}}\right)\left(\right.$ resp. $\left.\mathbb{k}_{2}=\mathbb{Q}\left(\sqrt{d_{2}}\right), \mathbb{k}_{3}=\mathbb{Q}\left(\sqrt{d_{3}}\right)\right), \mathbb{k}=\mathbb{k}_{3}(i), \mathbb{K}_{0}=\mathbb{k}_{1} \mathbb{k}_{2}$, $\mathbb{K}=\mathbb{K}_{0}(i), N_{1}$ (resp. $N_{2}, N_{3}$ ) la norme de $\mathbb{K}_{0} / \mathbb{k}_{1}$ (resp. $\mathbb{K}_{0} / \mathbb{K}_{2}, \mathbb{K}_{0} / \mathbb{K}_{3}$ ) et $\mathbf{E}_{\mathbb{k}}\left(\right.$ resp. $\left.\mathbf{E}_{\mathbb{K}_{0}}, \mathbf{E}_{\mathbb{K}}\right)$ le groupe des unités de $\mathbb{k}$ (resp. $\left.\mathbb{K}_{0}, \mathbb{K}\right)$.

On sait d'après [Kur-43] qu'un système fondamental d'unités (SFU) de $\mathbb{K}_{0}$ est, à une permutation près des indices, l'un des systèmes suivants :

(i) $\left\{\varepsilon_{1}, \varepsilon_{2}, \varepsilon_{3}\right\}$;

(ii) $\left\{\varepsilon_{1}, \varepsilon_{2}, \sqrt{\varepsilon_{3}}\right\}\left(N_{2}\left(\varepsilon_{3}\right)=1\right)$;

(iii) $\left\{\sqrt{\varepsilon_{1} \varepsilon_{2}}, \varepsilon_{2}, \varepsilon_{3}\right\}\left(N_{3}\left(\varepsilon_{1}\right)=N_{3}\left(\varepsilon_{2}\right)=1\right)$;

(iv) $\left\{\varepsilon_{1}, \sqrt{\varepsilon_{2}}, \sqrt{\varepsilon_{3}}\right\}\left(N_{1}\left(\varepsilon_{2}\right)=N_{1}\left(\varepsilon_{3}\right)=1\right)$;

(v) $\left\{\sqrt{\varepsilon_{1} \varepsilon_{2}}, \sqrt{\varepsilon_{2} \varepsilon_{3}}, \sqrt{\varepsilon_{1} \varepsilon_{3}}\right\}\left(N_{2}\left(\varepsilon_{3}\right)=N_{3}\left(\varepsilon_{j}\right)=1, j=1,2\right)$;

(vi) $\left\{\sqrt{\varepsilon_{1} \varepsilon_{2} \varepsilon_{3}}, \varepsilon_{2}, \varepsilon_{3}\right\}\left(N_{3}\left(\varepsilon_{1}\right)=N_{3}\left(\varepsilon_{2}\right)=N_{2}\left(\varepsilon_{3}\right)= \pm 1\right)$.

D'autre part, d'après [Az-93] ou [Az-99:2], on a les résultats suivants :

$\mathrm{R} 1: S F U$ de $\mathbb{k}=\mathbb{Q}(\sqrt{d}, i)$ où d est un entier naturel différent de 2 et sans facteurs carrés. Soit $\varepsilon_{0}=s+t \sqrt{d}$ l'unité fondamentale de $\mathbb{Q}(\sqrt{d})$.

(i) Si $\varepsilon_{0}$ est de norme -1 , alors $\left\{\varepsilon_{0}\right\}$ est un SFU de $\mathbb{Q}(\sqrt{d}, i)$. 
(ii) $\mathrm{Si} \varepsilon_{0}$ est de norme 1 , alors $\left\{\sqrt{i \varepsilon_{0}}\right\}$ est un SFU de $\mathbb{k}$ si et seulement si $s \pm 1$ est un carré dans $\mathbb{N}$ (i.e. si et seulement si $2 \varepsilon_{0}$ est un carré dans $\mathbb{Q}(\sqrt{d})$ ). Dans le cas contraire, $\left\{\varepsilon_{0}\right\}$ est un SFU de $\mathbb{k}$ (ce résultat se trouve aussi dans [Kub-56]).

$\mathrm{R} 2: S F U$ de $\mathbb{K}$. Soient $n$ un entier supérieur ou égal à 2 et $\xi_{n}$ une racine primitive $2^{n}$-ième de l'unité. Alors

$$
\begin{aligned}
\xi_{n}=\frac{1}{2}\left(\mu_{n}+\lambda_{n} i\right), \quad \text { où } \quad \mu_{n} & =\sqrt{2+\mu_{n-1}}, \lambda_{n}=\sqrt{2-\mu_{n-1}}, \\
\mu_{2} & =0, \lambda_{2}=2 \quad \text { et } \mu_{3}=\lambda_{3}=\sqrt{2} .
\end{aligned}
$$

De plus, soient $n_{0}$ le plus grand entier tel que $\xi_{n_{0}}$ appartient à $\mathbb{K},\left\{\varepsilon_{1}^{\prime}, \varepsilon_{2}^{\prime}, \varepsilon_{3}^{\prime}\right\}$ un SFU de $\mathbb{K}_{0}$ et $\varepsilon$ une unité de $\mathbb{K}_{0}$ telle que $\left(2+\mu_{n_{0}}\right) \varepsilon$ est un carré dans $\mathbb{K}_{0}$ (si elle existe). Alors, d'après [Az-93], l'un des systèmes suivants est un SFU de $\mathbb{K}$ :

(a) $\left\{\varepsilon_{1}^{\prime}, \varepsilon_{2}^{\prime}, \varepsilon_{3}^{\prime}\right\}$ si $\varepsilon$ n'existe pas,

(b) $\left\{\varepsilon_{1}^{\prime}, \varepsilon_{2}^{\prime}, \sqrt{\xi_{n_{0}} \varepsilon}\right\}$ si $\varepsilon$ existe; dans ce cas on a $\varepsilon=\varepsilon_{1}^{\prime i_{1}} \varepsilon_{2}^{\prime} i_{2} \varepsilon_{3}^{\prime}$, où $i_{1}, i_{2} \in$ $\{0,1\}$ (à une permutation près).

\subsection{SFU de certains corps de nombres de degré 4 ou 8}

LEMME 5. Soient $d$ un entier relatif sans facteur carré et $\varepsilon=x+y \sqrt{d}$ l'unité fondamentale de $\mathbb{Q}(\sqrt{d})$ où $x$ et y sont des entiers ou bien des demientiers. On suppose que $\varepsilon$ est de norme 1 . Alors $2(x \pm 1)$ et $2 d(x \pm 1)$ ne sont pas des carrés dans $\mathbb{Q}$.

Preuve. Comme

$$
\sqrt{\varepsilon}=\frac{1}{2}(\sqrt{2(x+1)}+\sqrt{2(x-1)}) \quad \text { et } \quad \sqrt{2(x+1)} \sqrt{2(x-1)}=2 y \sqrt{d},
$$

alors $\sqrt{2(x-1)}$ et $\sqrt{2(x+1)}$ ne sont pas des carrés dans $\mathbb{Q}$. Si $2 d(x \pm 1)=$ $s^{2}$, alors $\sqrt{2(x \pm 1)}=(s / d) \sqrt{d}$ appartient à $\mathbb{Q}(\sqrt{d})$ et $\sqrt{2(x \mp 1)}=2 y d / s$ appartient à $\mathbb{Q}$. Par suite $\sqrt{\varepsilon} \in \mathbb{Q}(\sqrt{d})$, donc on a une contradiction.

Lemme 6. Soient $q$ un nombre premier impair congru $\grave{a}-1$ modulo 4 et $\varepsilon=x+y \sqrt{q}$ l'unité fondamentale de $\mathbb{Q}(\sqrt{q})$. Alors $x$ est un entier naturel pair, $x \pm 1$ est un carré dans $\mathbb{N}$ et $2 \varepsilon$ est un carré dans $\mathbb{Q}(\sqrt{q})$.

Preuve. Comme $q \equiv-1 \bmod 4$, alors $\varepsilon=x+y \sqrt{q}$ est tel que $(x, y) \in$ $\mathbb{Z}^{2}$ et $x^{2}-q y^{2}=1$. D'où $(x+1)(x-1)=q y^{2}$. Du fait que $(x+1)-(x-1)=2$, le plus grand commun diviseur de $x+1$ et $x-1$ est un diviseur de 2. Par suite il existe $\left(y_{1}, y_{2}\right) \in \mathbb{Z}^{2}$ tel que

$$
\left\{\begin{array}{l}
x-1=q^{i} 2^{j} y_{1}^{2} \\
x+1=q^{i^{\prime}} 2^{j} y_{2}^{2}
\end{array}\right.
$$

où $i, i^{\prime}, j \in\{0,1\}, i+i^{\prime}=1$ et $2^{j} y_{1} y_{2}=y$. 
Si $x-1$ est pair, alors $j=1$ et $\sqrt{2(x \mp 1)}$ est un carré dans $\mathbb{N}$. D'après le lemme 5, ceci est impossible. Par conséquent, $x$ est pair et $x \pm 1$ est un carré dans $\mathbb{N}$. De plus, comme $x-1$ est impair, alors $j=0$ et $2 \varepsilon$ est un carré dans $\mathbb{Q}(\sqrt{q})$.

LeMme 7. Soient $p$ un nombre premier impair et $\varepsilon=x+y \sqrt{2 p}$ l'unité fondamentale de $\mathbb{Q}(\sqrt{2 p})$. On suppose que $\varepsilon$ est de norme 1 . Alors $x \pm 1$ est un carré dans $\mathbb{N}$ et $2 \varepsilon$ est un carré dans $\mathbb{Q}(\sqrt{2 p})$.

Preuve. On suppose que $x \pm 1$ n'est pas un carré dans $\mathbb{N}$. Comme $\varepsilon=x+y \sqrt{2 p}$ est de norme 1 , alors $(x+1)(x-1)=2 p y^{2}$. Les entiers $x+1$ et $x-1$ ont pour diviseurs communs les diviseurs de 2 . Par suite on a :

$$
\left\{\begin{array} { r l } 
{ x + 1 } & { = 2 y _ { 1 } ^ { 2 } , } \\
{ x - 1 } & { = p y _ { 2 } ^ { 2 } , } \\
{ y } & { = y _ { 1 } y _ { 2 } , }
\end{array} \quad \text { ou } \quad \left\{\begin{array}{rl}
x-1 & =2 y_{1}^{2}, \\
x+1 & =p y_{2}^{2}, \\
y & =y_{1} y_{2} .
\end{array}\right.\right.
$$

Donc $\sqrt{2(x \mp 1)}$ est un carré dans $\mathbb{N}$. D'après le lemme 5 , ceci est impossible. Il vient donc que $x \pm 1$ est un carré dans $\mathbb{N}$. De plus, d'après le résultat R1, $x \pm 1$ est un carré dans $\mathbb{N}$ si et seulement si $2 \varepsilon$ est un carré dans $\mathbb{Q}(\sqrt{2 p})$. Ceci termine la preuve.

Lemme 8. Soient $p$ et $q$ nombres premiers impairs, $\mathbb{K}_{0}=\mathbb{Q}(\sqrt{q}, \sqrt{2 p})$, $\varepsilon_{1}$ (resp. $\left.\varepsilon_{2}, \varepsilon_{3}\right)$ l'unité fondamentale de $\mathbb{k}_{1}=\mathbb{Q}(\sqrt{q})$ (resp. $\mathbb{k}_{2}=\mathbb{Q}(\sqrt{2 p})$, $\left.\mathbb{k}_{3}=\mathbb{Q}(\sqrt{2 p q})\right)$. On suppose que toutes les unités $\varepsilon_{i}$ sont de norme 1 et que $2 \varepsilon_{3}$ n'est pas un carré dans $\mathbb{k}_{3}$. On pose $\varepsilon_{3}=x+y \sqrt{2 p q}$. Alors on $a$ :

(i) Si $2 p(x \pm 1)$ est un carré dans $\mathbb{N}$, alors $2 \varepsilon_{3}$ est un carré dans $\mathbb{K}_{0}$ et $\left\{\sqrt{\varepsilon_{1} \varepsilon_{3}}, \sqrt{\varepsilon_{1} \varepsilon_{2}}, \sqrt{\varepsilon_{2} \varepsilon_{3}}\right\}$ est un $S F U$ de $\mathbb{K}_{0}$.

(ii) Si $2 q(x \pm 1)$ est un carré dans $\mathbb{N}$, alors $\varepsilon_{3}$ est un carré dans $\mathbb{K}_{0}$ et $\left\{\varepsilon_{1}, \sqrt{\varepsilon_{1} \varepsilon_{2}}, \sqrt{\varepsilon_{3}}\right\}$ est un $S F U$ de $\mathbb{K}_{0}$.

Preuve. Soit $\varepsilon_{3}=x+y \sqrt{2 p q}$ tel que $(x-1)(x+1)=2 p q y^{2}$. On sait que $2 \varepsilon_{3}$ est un carré dans $\mathbb{k}_{3}$ si et seulement si $x \pm 1$ est un carré dans $\mathbb{N}$ (voir résultat $\mathrm{R} 1$ ).

D'après le lemme $5,2(x \pm 1)$ et $p q(x \pm 1)$ ne sont pas des carrés dans $\mathbb{N}$. Ainsi on a :

- Si $2 p(x \pm 1)$ est un carré dans $\mathbb{N}$, alors $q(x \mp 1)$ est un carré dans $\mathbb{N}$ et il existe $\left(y_{1}, y_{2}\right) \in \mathbb{Z}^{2}$ tel que

$$
\left\{\begin{array} { l } 
{ x \pm 1 = 2 p y _ { 1 } ^ { 2 } , } \\
{ x \mp 1 = q y _ { 2 } ^ { 2 } , }
\end{array} \quad \left\{\begin{array}{l}
\sqrt{2 \varepsilon_{3}}=y_{1} \sqrt{2 p}+y_{2} \sqrt{q}, \\
\sqrt{\varepsilon_{3}}=\frac{1}{2}\left(2 y_{1} \sqrt{p}+y_{2} \sqrt{2 q}\right) .
\end{array}\right.\right.
$$

- Si $2 q(x \pm 1)$ est un carré dans $\mathbb{N}$, alors $p(x \mp 1)$ est un carré dans $\mathbb{N}$ et il existe $\left(y_{1}, y_{2}\right) \in \mathbb{Z}^{2}$ tel que

$$
\left\{\begin{array} { l } 
{ x \pm 1 = 2 q y _ { 1 } ^ { 2 } , } \\
{ x \mp 1 = p y _ { 2 } ^ { 2 } , }
\end{array} \quad \left\{\begin{array}{l}
\sqrt{2 \varepsilon_{3}}=y_{1} \sqrt{2 q}+y_{2} \sqrt{p}, \\
\sqrt{\varepsilon_{3}}=\frac{1}{2}\left(2 y_{1} \sqrt{q}+y_{2} \sqrt{2 p}\right) .
\end{array}\right.\right.
$$


Par conséquent, si $2 p(x \pm 1)$ est un carré dans $\mathbb{N}$, alors $2 \varepsilon_{3}$ est un carré dans $\mathbb{K}_{0}$ et si $2 q(x \pm 1)$ est un carré dans $\mathbb{N}$, alors $\varepsilon_{3}$ est un carré dans $\mathbb{K}_{0}$. D'autre part, d'après les lemmes 6 et $7,2 \varepsilon_{1}$ et $2 \varepsilon_{2}$ sont des carrés dans $\mathbb{K}_{0}$. D'où $\varepsilon_{1} \varepsilon_{2}$ est un carré dans $\mathbb{K}_{0}$. De même, si $2 \varepsilon_{3}$ est un carré dans $\mathbb{K}_{0}$, alors $\varepsilon_{1} \varepsilon_{3}$ et $\varepsilon_{2} \varepsilon_{3}$ sont des carrés dans $\mathbb{K}_{0}$ et donc $\left\{\sqrt{\varepsilon_{1} \varepsilon_{2}}, \sqrt{\varepsilon_{1} \varepsilon_{3}}, \sqrt{\varepsilon_{2} \varepsilon_{3}}\right\}$ est un SFU de $\mathbb{K}_{0}$. Dans le cas où $\varepsilon_{3}$ est un carré dans $\mathbb{K}_{0}$, l'unité $\varepsilon_{1} \varepsilon_{2} \varepsilon_{3}$ est un carré dans $\mathbb{K}_{0}$ et d'après les résultats de [Kur-43], rappelés au début de ce paragraphe, $\left\{\varepsilon_{1}, \sqrt{\varepsilon_{1} \varepsilon_{2}}, \sqrt{\varepsilon_{3}}\right\}$ est un $\mathrm{SFU}$ de $\mathbb{K}_{0}$.

ThÉORÈme 9 . On se place dans les conditions du lemme 8. Soit $\mathbb{K}=$ $\mathbb{K}_{0}(i)$. Alors on $a$ :

(i) Si $2 p(x \pm 1)$ est un carré dans $\mathbb{N}$, alors $\left\{\sqrt{i \varepsilon_{1}}, \sqrt{i \varepsilon_{2}}, \sqrt{i \varepsilon_{3}}\right\}$ est un $S F U$ de $\mathbb{K}$.

(ii) Si $2 q(x \pm 1)$ est un carré dans $\mathbb{N}$, alors $\left\{\sqrt{i \varepsilon_{1}}, \sqrt{\varepsilon_{3}}, \sqrt{i \varepsilon_{2}}\right\}$ est un $S F U$ de $\mathbb{K}$.

Preuve. Les unités $\varepsilon_{1}$ et $\varepsilon_{2}$ jouent un rôle symétrique.

(i) Soit $\varepsilon=\sqrt{\varepsilon_{1} \varepsilon_{2}} \sqrt{\varepsilon_{1} \varepsilon_{3}} \sqrt{\varepsilon_{2} \varepsilon_{3}}=\varepsilon_{1}\left(\sqrt{\varepsilon_{2} \varepsilon_{3}}\right)^{2}$. On sait que $\sqrt{i \varepsilon} \in \mathbb{K}$ si et seulement si $\sqrt{2 \varepsilon} \in \mathbb{K}_{0}(\operatorname{car} \sqrt{i}=(1+i) / \sqrt{2})$. Comme $2 \varepsilon_{1}$ est un carré dans $\mathbb{K}_{0}$, alors $2 \varepsilon$ est un carré dans $\mathbb{K}_{0}$. Un SFU de $\mathbb{K}_{0}$ est donné par le lemme précédent. D'où, d'après le résultat $\mathrm{R} 2,\left\{\sqrt{\varepsilon_{1} \varepsilon_{2}}, \sqrt{\varepsilon_{1} \varepsilon_{3}}, \sqrt{i \varepsilon_{1} \varepsilon_{2} \varepsilon_{3}}\right\}$ est un SFU de $\mathbb{K}$. Comme $2 \varepsilon_{i}$ est un carré dans $\mathbb{K}_{0}$ pour $i=1,2,3$, alors $\left\{\sqrt{i \varepsilon_{1}}, \sqrt{i \varepsilon_{2}}, \sqrt{i \varepsilon_{3}}\right\}$ est aussi un SFU de $\mathbb{K}$.

(ii) Soit $\varepsilon=\varepsilon_{2}$. On sait d'après le lemme 7 que $2 \varepsilon_{2}$ est un carré dans $\mathbb{K}_{0}$ et le lemme 8 nous donne un SFU de $\mathbb{K}_{0}$. Donc en utilisant le résultat $\mathrm{R} 2$, on trouve que $\left\{\sqrt{\varepsilon_{1} \varepsilon_{2}}, \sqrt{\varepsilon_{3}}, \sqrt{i \varepsilon_{2}}\right\}$ est un SFU de $\mathbb{K}$. Puisque $2 \varepsilon_{i}$ est un carré dans $\mathbb{K}_{0}$ pour $i=1,2$, alors $\left\{\sqrt{i \varepsilon_{1}}, \sqrt{\varepsilon_{3}}, \sqrt{i \varepsilon_{2}}\right\}$ est aussi un SFU de $\mathbb{K}$.

ThÉORÈME 10. Soient $p$ et $q$ deux nombres premiers impairs tels que $p \equiv$ $-q \equiv 1 \bmod 4, \mathbb{K}_{0}=\mathbb{Q}(\sqrt{p}, \sqrt{2 q})$ et $\varepsilon_{1}$ (resp. $\left.\varepsilon_{2}, \varepsilon_{3}\right)$ l'unité fondamentale de $\mathbb{k}_{1}=\mathbb{Q}(\sqrt{p})\left(\right.$ resp. $\left.\mathbb{k}_{2}=\mathbb{Q}(\sqrt{2 q}), \mathbb{k}_{3}=\mathbb{Q}(\sqrt{2 p q})\right)$. On pose $\varepsilon_{3}=x+y \sqrt{2 p q}$ et on suppose que $2 \varepsilon_{3}$ n'est pas un carré dans $\mathbb{k}_{3}$. Alors on $a$ :

(i) $\left\{\varepsilon_{1}, \varepsilon_{2}, \sqrt{\varepsilon_{3}}\right\}$ est un $S F U$ de $\mathbb{K}_{0} \Leftrightarrow 2 p(x \pm 1)$ est un carré dans $\mathbb{N}$. Dans ce cas $\left\{\varepsilon_{1}, \sqrt{\varepsilon_{3}}, \sqrt{i \varepsilon_{2}}\right\}$ est un $S F U$ de $\mathbb{K}=\mathbb{K}_{0}(i)$.

(ii) $\left\{\varepsilon_{1}, \varepsilon_{2}, \sqrt{\varepsilon_{2} \varepsilon_{3}}\right\}$ est un $S F U$ de $\mathbb{K}_{0} \Leftrightarrow 2 q(x \pm 1)$ est un carré dans $\mathbb{N}$. Dans ce cas $\left\{\varepsilon_{1}, \sqrt{\varepsilon_{2} \varepsilon_{3}}, \sqrt{i \varepsilon_{2}}\right\}$ est un $S F U$ de $\mathbb{K}=\mathbb{K}_{0}(i)$.

Preuve. Les unités $\varepsilon_{1}$ et $\varepsilon_{2}$ ne jouent pas un rôle symétrique dans ce théorème. On a que $\varepsilon_{1}$ est de norme $-1, \varepsilon_{2}$ et $\varepsilon_{3}$ sont de norme 1 . D'après le lemme $7,2 \varepsilon_{2}$ est un carré dans $\mathbb{k}_{2}$. D'où $\varepsilon_{2}$ n'est pas un carré dans $\mathbb{K}$, car sinon $\sqrt{2} \in \mathbb{K}_{0}$. Soit $\varepsilon_{3}=x+y \sqrt{2 p q}$. Par hypothèse $2 \varepsilon_{3}$ n'est pas un carré dans $\mathbb{k}_{3}$, ce qui est équivalent au fait que $x \pm 1$ n'est pas un carré dans $\mathbb{N}$ (le résultat $\mathrm{R} 1$ ). De la même façon que dans le lemme 8 , comme $(x+1)(x-1)=2 p q y^{2}$, alors $2 p(x \pm 1)$ ou $2 q(x \pm 1)$ est un carré dans $\mathbb{N}$. 
(a) On suppose que $2 p(x \pm 1)$ est un carré dans $\mathbb{N}$. Si $2 p(x-1)$ est un carré dans $\mathbb{N}$, alors il existe $\left(y_{1}, y_{2}\right) \in \mathbb{Z}^{2}$ tel que

$$
\left\{\begin{array} { l } 
{ x - 1 = 2 p y _ { 1 } ^ { 2 } , } \\
{ x + 1 = q y _ { 2 } ^ { 2 } , }
\end{array} \quad \left\{\begin{array}{rl}
\sqrt{2 \varepsilon_{3}} & =y_{1} \sqrt{2 p}+y_{2} \sqrt{q}, \\
\sqrt{\varepsilon_{3}} & =\frac{1}{2}\left(2 y_{1} \sqrt{p}+y_{2} \sqrt{2 q}\right) .
\end{array}\right.\right.
$$

Il en est de même si $2 p(x+1)$ est un carré dans $\mathbb{N}$. Il vient donc que si $2 p(x \pm 1)$ est un carré dans $\mathbb{N}$, alors $\sqrt{\varepsilon_{3}} \in \mathbb{K}_{0}$ et $\sqrt{2 \varepsilon_{3}} \notin \mathbb{K}_{0}$.

(b) On fait la même chose si $2 q(x \pm 1)$ est un carré dans $\mathbb{N}$ et on trouve que $\sqrt{\varepsilon_{3}} \notin \mathbb{K}_{0}$ et $\sqrt{2 \varepsilon_{3}} \in \mathbb{K}_{0}$. Si $\sqrt{2 \varepsilon_{3}} \in \mathbb{K}$, alors $\sqrt{\varepsilon_{2} \varepsilon_{3}} \in \mathbb{K}_{0}$, puisque $\sqrt{2 \varepsilon_{2}} \in \mathbb{K}$. Ainsi on a :

(i) $\left\{\varepsilon_{1}, \varepsilon_{2}, \sqrt{\varepsilon_{3}}\right\}$ est un SFU de $\mathbb{K}_{0}$ si et seulement si $2 p(x \pm 1)$ est un carré dans $\mathbb{N}$.

(ii) $\left\{\varepsilon_{1}, \varepsilon_{2}, \sqrt{\varepsilon_{2} \varepsilon_{3}}\right\}$ est un SFU de $\mathbb{K}_{0}$ si et seulement si $2 q(x \pm 1)$ est un carré dans $\mathbb{N}$.

D'autre part, $2 \varepsilon_{2}$ est un carré dans $\mathbb{k}_{2}$ entraîne que $\sqrt{i \varepsilon_{2}} \in \mathbb{k}_{2}(i)$. En utilisant le résultat $\mathrm{R} 2$, on trouve qu'un $\mathrm{SFU}$ de $\mathbb{K}=\mathbb{K}_{0}(i)$ est :

(i') $\left\{\varepsilon_{1}, \sqrt{i \varepsilon_{2}}, \sqrt{\varepsilon_{3}}\right\}$ si $2 p(x \pm 1)$ est un carré dans $\mathbb{N}$.

(ii') $\left\{\varepsilon_{1}, \sqrt{i \varepsilon_{2}}, \sqrt{\varepsilon_{2} \varepsilon_{3}}\right\}$ si $2 q(x \pm 1)$ est un carré dans $\mathbb{N}$.

ThÉORÈme 11. Soient $\mathbb{K}_{0}=\mathbb{Q}(\sqrt{q}, \sqrt{2 p}), \mathbb{K}=\mathbb{K}_{0}(i), \mathbb{k}_{1}=\mathbb{Q}(\sqrt{q})$, $\mathbb{k}_{2}=\mathbb{Q}(\sqrt{2 p})$ et $\mathbb{k}_{3}=\mathbb{Q}(\sqrt{2 p q})$. On garde les autres notations du théorème précédent. On suppose que $\varepsilon_{2}$ est de norme -1 et $2 \varepsilon_{3}$ n'est pas un carré dans $\mathbb{k}_{3}$. Alors si $\varepsilon_{3}=x+y \sqrt{2 p q}$ on $a$ :

(i) $\left\{\varepsilon_{1}, \varepsilon_{2}, \sqrt{\varepsilon_{3}}\right\}$ est un $S F U$ de $\mathbb{K}_{0}$ si et seulement si $2 q(x \pm 1)$ est un carré dans $\mathbb{N}$. Dans ce cas un $S F U$ de $\mathbb{K}$ est $\left\{\varepsilon_{2}, \sqrt{i \varepsilon_{1}}, \sqrt{\varepsilon_{3}}\right\}$.

(ii) $\left\{\varepsilon_{1}, \varepsilon_{2}, \sqrt{\varepsilon_{1} \varepsilon_{3}}\right\}$ est un $S F U$ de $\mathbb{K}_{0}$ si et seulement si $2 p(x \pm 1)$ est un carré dans $\mathbb{N}$. Dans ce cas, un $S F U$ de $\mathbb{K}$ est $\left\{\varepsilon_{2}, \sqrt{i \varepsilon_{1}}, \sqrt{\varepsilon_{1} \varepsilon_{3}}\right\}$.

Preuve. Il suffit de remarquer que $2 \varepsilon_{1}$ est un carré dans $\mathbb{k}_{1}, \varepsilon_{1}$ n'est pas un carré dans $\mathbb{K}_{0}$ et si $\varepsilon_{3}=x+y \sqrt{2 p q}$, alors on a :

(i) $2 q(x \pm 1)$ est un carré dans $\mathbb{N} \Leftrightarrow \sqrt{\varepsilon_{3}} \in \mathbb{K}_{0}$ et $\sqrt{2 \varepsilon_{3}} \notin \mathbb{K}_{0}$.

(ii) $2 p(x \pm 1)$ est un carré dans $\mathbb{N} \Leftrightarrow \sqrt{2 \varepsilon_{3}} \in \mathbb{K}_{0}$ et $\sqrt{\varepsilon_{3}} \notin \mathbb{K}_{0}$.

Avec le même raisonnement que dans le théorème précédent, on arrive aux résultats voulus.

ThÉORÈmE 12. Soient $p$ et $q$ deux nombres premiers impairs tels que $q \equiv-1 \bmod 4, \mathbb{K}_{0}=\mathbb{Q}(\sqrt{2}, \sqrt{p q}), \mathbb{K}=\mathbb{K}_{0}(i)$ et $\varepsilon_{1}$ (resp. $\left.\varepsilon_{2}, \varepsilon_{3}\right)$ l'unité fondamentale de $\mathbb{k}_{1}=\mathbb{Q}(\sqrt{2})$ (resp. $\mathbb{k}_{2}=\mathbb{Q}(\sqrt{p q}), \mathbb{k}_{3}=\mathbb{Q}(\sqrt{2 p q})$ ). On suppose que $2 \varepsilon_{3}$ n'est pas un carré dans $\mathbb{k}_{3}$.

1. Si $\varepsilon_{2}=x+y \sqrt{p q}$ avec $(x, y) \in \mathbb{Z}^{2}$. Alors on $a$ : 
(i) Si $x \pm 1$ est un carré dans $\mathbb{N}$, alors $\left\{\varepsilon_{1}, \sqrt{\varepsilon_{2}}, \varepsilon_{3}\right\}$ est un $S F U$ de $\mathbb{K}_{0}$ et de $\mathbb{K}$.

(ii) Sinon, $\left\{\varepsilon_{1}, \varepsilon_{2}, \sqrt{\varepsilon_{2} \varepsilon_{3}}\right\}$ est un $S F U$ de $\mathbb{K}_{0}$ et de $\mathbb{K}$.

2. Si $\varepsilon_{2}=x / 2+(y / 2) \sqrt{p q}$ avec $x$ et $y$ deux entiers impairs. Alors les deux corps $\mathbb{K}_{0}$ et $\mathbb{K}$ ont le même $S F U\left\{\varepsilon_{1}, \varepsilon_{2}, \sqrt{\varepsilon_{2} \varepsilon_{3}}\right\}$.

Preuve. 1 . On a que $\varepsilon_{1}$ est de norme $-1, \varepsilon_{2}$ et $\varepsilon_{3}$ sont de norme 1 et $(x-1)(x+1)=p q y^{2}$. D'après le lemme $5,2 p q(x \pm 1)$ n'est pas un carré dans $\mathbb{N}$. Ainsi on a :

(a) Soit $p q(x \pm 1)$ un carré dans $\mathbb{N}$. Si $p q(x-1)$ est un carré dans $\mathbb{N}$, alors il existe $\left(y_{1}, y_{2}\right) \in \mathbb{Z}^{2}$ tel que

$$
\left\{\begin{array}{l}
x-1=p q y_{1}^{2}, \quad \sqrt{\varepsilon_{2}}=\left(y_{1} \sqrt{2 p q}+y_{2} \sqrt{2}\right) / 2 \in \mathbb{K}_{0} . \\
x+1=y_{2}^{2},
\end{array}\right.
$$

Il en est de même si $p q(x+1)$ est un carré dans $\mathbb{N}$.

(b) Soit $p(x \pm 1)$ ou $2 p(x \pm 1)$ un carré dans $\mathbb{N}$. Si $p(x-1)$ est un carré dans $\mathbb{N}$, alors il existe $\left(y_{1}, y_{2}\right) \in \mathbb{Z}^{2}$ tel que

$$
\left\{\begin{array}{l}
x-1=p y_{1}^{2}, \quad \sqrt{\varepsilon_{2}}=\left(y_{1} \sqrt{2 p}+y_{2} \sqrt{2 q}\right) / 2 \notin \mathbb{K}_{0}, \sqrt{p \varepsilon_{2}} \text { et } \sqrt{q \varepsilon_{2}} \in \mathbb{K}_{0} . \\
x+1=q y_{2}^{2},
\end{array}\right.
$$

Dans les trois cas qui restent on a que $\sqrt{\varepsilon_{2}} \notin \mathbb{K}_{0}, \sqrt{p \varepsilon_{2}}$ et $\sqrt{q \varepsilon_{2}} \in \mathbb{K}_{0}$.

Soit $\varepsilon_{3}=s+t \sqrt{2 p q}$. On a $(s-1)(s+1)=2 p q t^{2}$. Comme $2 \varepsilon_{3}$ n'est pas un carré dans $\mathbb{k}_{3}$, alors $s \pm 1$ n'est pas un carré dans $\mathbb{N}$. Avec un raisonnement semblable à celui fait pour $\varepsilon_{2}$, on trouve que les seuls cas possibles sont :

- $2 q(s \pm 1)$ est un carré dans $\mathbb{N}$;

- $2 p(s \pm 1)$ est un carré dans $\mathbb{N}$.

Dans ces deux cas, $\sqrt{\varepsilon_{3}} \notin \mathbb{K}_{0}$ et $\sqrt{p \varepsilon_{3}}, \sqrt{q \varepsilon_{3}}$ appartiennent à $\mathbb{K}_{0}$. Si $\sqrt{p \varepsilon_{2}} \in$ $\mathbb{K}_{0}$ et $\sqrt{p \varepsilon_{3}} \in \mathbb{K}_{0}$, alors $\sqrt{\varepsilon_{2} \varepsilon_{3}} \in \mathbb{K}_{0}$.

Enfin, avec toutes ces données et les résultats de [Kur-43] rappelés au début de ce paragraphe on conclut que :

(i) Si $x \pm 1$ est un carré dans $\mathbb{N}$, alors $\left\{\varepsilon_{1}, \sqrt{\varepsilon_{2}}, \varepsilon_{3}\right\}$ est un SFU de $\mathbb{K}_{0}$.

(ii) Sinon, $\left\{\varepsilon_{1}, \varepsilon_{2}, \sqrt{\varepsilon_{2} \varepsilon_{3}}\right\}$ est un SFU de $\mathbb{K}_{0}$.

D'autre part, d'après le théorème 2 (ii) sur les unités de $\mathbb{Q}(\sqrt{d}, \sqrt{2}, i)$ de [H-Y-90] une condition suffisante pour qu'un SFU de $\mathbb{K}_{0}$ soit aussi un SFU de $\mathbb{K}$, est que $s \pm 1$ ou $x \pm 1$ n'est pas un carré dans $\mathbb{N}$. Or, dans notre cas, on suppose que $2 \varepsilon_{3}$ n'est pas un carré dans $\mathbb{k}_{3}$, ce qui est équivalent au fait que $s \pm 1$ n'est pas un carré dans $\mathbb{N}$. D'où $\mathbb{K}_{0}$ et $\mathbb{K}$ ont un même SFU.

2. Soit $\varepsilon_{2}=x / 2+(y / 2) \sqrt{p q}$. Comme $\varepsilon_{2}$ est de norme 1 , alors on a $(x-2)(x+2)=p q y^{2},(x+2)-(x-2)=4, x-2$ et $x+2$ sont impairs. Par suite $x+2$ et $x-2$ sont premiers entre eux. 
(a) Soit $p q(x \pm 2)$ un carré dans $\mathbb{N}$. Si $p q(x-2)$ est un carré dans $\mathbb{N}$, alors il existe $\left(y_{1}, y_{2}\right) \in \mathbb{Z}^{2}$ tel que

$$
\left\{\begin{array}{l}
x-2=p q y_{1}^{2}, \\
x+2=y_{2}^{2},
\end{array} \quad \sqrt{\varepsilon_{2}}=\left(y_{1} \sqrt{p q}+y_{2}\right) / 2 \in \mathbb{k}_{3} .\right.
$$

Ceci est absurde puisque $\varepsilon_{2}$ est l'unité fondamentale de $\mathbb{k}_{2}=\mathbb{Q}(\sqrt{p q})$. Il en est de même si $p q(x+2)$ est un carré dans $\mathbb{N}$. Donc ces deux cas ne se présentent pas.

(b) Soit $p(x \pm 2)$ un carré dans $\mathbb{N}$. Si $p(x-2)$ est un carré dans $\mathbb{N}$, alors il existe $\left(y_{1}, y_{2}\right) \in \mathbb{Z}^{2}$ tel que

$$
\left\{\begin{array}{l}
x-2=p y_{1}^{2}, \\
x+2=q y_{2}^{2},
\end{array} \quad \sqrt{\varepsilon_{2}}=\left(y_{1} \sqrt{p}+y_{2} \sqrt{q}\right) / 2 \notin \mathbb{K}_{0}, \sqrt{p \varepsilon_{2}} \text { et } \sqrt{q \varepsilon_{2}} \in \mathbb{K}_{0} .\right.
$$

Les résultats du cas précédent concernant $\varepsilon_{3}$ restent valables ici, c'est-à-dire $\sqrt{\varepsilon_{3}} \notin \mathbb{K}_{0}, \sqrt{p \varepsilon_{3}}$ et $\sqrt{q \varepsilon_{3}} \in \mathbb{K}_{0}$. Par conséquent, $\sqrt{\varepsilon_{2} \varepsilon_{3}} \in \mathbb{K}_{0}$. De la même façon que dans le premier cas on conclut que $\left\{\varepsilon_{1}, \varepsilon_{2}, \sqrt{\varepsilon_{2} \varepsilon_{3}}\right\}$ est un SFU de $\mathbb{K}_{0}$ et $\mathbb{K}$.

Remarque 13. On garde les notations précédentes. Soient $d=p q, \varepsilon_{1}$ l'unité fondamentale de $\mathbb{Q}(\sqrt{d}), \varepsilon_{2}$ l'unité fondamentale de $\mathbb{Q}(\sqrt{2 d})$ et $\Delta_{i}$ la partie sans carré de $N\left(\varepsilon_{i}+1\right)$. On trouve dans [H-Y-90] que si $Q=2$ alors $\Delta_{1}=\Delta_{2}=2$ ou bien $\Delta_{1}=2 d$ et $\Delta_{2}=d$. Or si $\varepsilon_{1}=x+y \sqrt{d}$ et $\varepsilon_{2}=s+t \sqrt{2 d}$, alors

$$
\begin{array}{lll}
(x-1)(x+1)=d y^{2} & \text { et } & N\left(\varepsilon_{1}+1\right)=2(x+1) ; \\
(s-1)(s+1)=2 d t^{2} & \text { et } & N\left(\varepsilon_{2}+1\right)=2(s+1) ;
\end{array}
$$

et

$\Delta_{1}=2 \Leftrightarrow(x+1)$ est un carré dans $\mathbb{N} \Leftrightarrow d(x-1)$ est un carré dans $\mathbb{N}$. $\Delta_{1}=2 d \Leftrightarrow d(x+1)$ est un carré dans $\mathbb{N} \Leftrightarrow(x-1)$ est un carré dans $\mathbb{N}$. $\Delta_{2}=2 \Leftrightarrow(s+1)$ est un carré dans $\mathbb{N} \Leftrightarrow 2 d(s-1)$ est un carré dans $\mathbb{N}$. $\Delta_{2}=d \Leftrightarrow 2 d(s+1)$ est un carré dans $\mathbb{N} \Leftrightarrow(s-1)$ est un carré dans $\mathbb{N}$.

Par suite si $x \pm 1$ ou $s \pm 1$ n'est pas un carré dans $\mathbb{N}$ alors $Q=1$.

THÉORÈME 14. Soient $p$ et $q$ deux nombres premiers impairs tels que $p \equiv$ $-q \equiv 1 \bmod 4, \mathbb{K}_{0}=\mathbb{Q}(\sqrt{p}, \sqrt{q}), \mathbb{K}=\mathbb{K}_{0}(i)$ et $\varepsilon_{1}$ (resp. $\left.\varepsilon_{2}, \varepsilon_{3}\right)$ l'unité fondamentale de $\mathbb{Q}(\sqrt{p})$ (resp. $\mathbb{Q}(\sqrt{q}), \mathbb{Q}(\sqrt{p q})$ ). On suppose que $2 \varepsilon_{3}$ est un carré dans $\mathbb{Q}(\sqrt{p q})$. Alors $\left\{\varepsilon_{1}, \varepsilon_{2}, \sqrt{\varepsilon_{2} \varepsilon_{3}}\right\}$ est un $S F U$ de $\mathbb{K}_{0}$ et $\left\{\varepsilon_{1}, \sqrt{i \varepsilon_{2}}, \sqrt{\varepsilon_{2} \varepsilon_{3}}\right\}$ est un $S F U$ de $\mathbb{K}$.

Preuve. On sait, d'après le lemme 6 , que $2 \varepsilon_{2}$ est un carré dans $\mathbb{Q}(\sqrt{q})$. Comme $2 \varepsilon_{3}$ est un carré dans $\mathbb{Q}(\sqrt{p q})$, alors $\sqrt{\varepsilon_{2} \varepsilon_{3}}$ appartient à $\mathbb{K}_{0}$. De plus $\sqrt{\varepsilon_{2}} \notin \mathbb{K}_{0}$ et $\sqrt{\varepsilon_{3}} \notin \mathbb{K}_{0}$. D'où $\left\{\varepsilon_{1}, \varepsilon_{2}, \sqrt{\varepsilon_{2} \varepsilon_{3}}\right\}$ est un SFU de $\mathbb{K}_{0}$. D'autre part 
$\sqrt{2 \varepsilon_{2}} \in \mathbb{K}_{0} \Leftrightarrow \sqrt{i \varepsilon_{2}} \in \mathbb{K}$. Par suite, d'après le résultat $\mathrm{R} 2,\left\{\varepsilon_{1}, \sqrt{i \varepsilon_{2}}, \sqrt{\varepsilon_{2} \varepsilon_{3}}\right\}$ est un SFU de $\mathbb{K}$.

REMARQue 15. Soient $p$ et $q$ deux nombres premiers impairs tels que $p \equiv 1 \bmod 8,-q \equiv 1 \bmod 4$ et $\left(\frac{p}{q}\right)=-1$. Soit $\varepsilon_{3}$ l'unité fondamentale de $\mathbb{Q}(\sqrt{p q})$. Alors $2 \varepsilon_{3}$ est un carré dans $\mathbb{Q}(\sqrt{p q})$ (c.a.d. $\left.Q=2\right)$.

Preuve. Soit $\varepsilon_{3}=x+y \sqrt{p q}$. Comme $\varepsilon_{3}$ est de norme 1 , alors on a $(x-1)(x+1)=p q y^{2}$. D'après le lemme $5,2(x \pm 1)$ et $2 p q(x \pm 1)$ ne sont pas des carrés dans $\mathbb{N}$. Ainsi on a deux cas :

(a) $p(x-1)$ ou $2 p(x-1)$ est un carré dans $\mathbb{N}$. Si $p(x-1)$ est un carré dans $\mathbb{N}$, alors il existe $\left(y_{1}, y_{2}\right) \in \mathbb{Z}^{2}$ tel que

$$
\left\{\begin{array}{l}
x-1=p y_{1}^{2}, \\
x+1=q y_{2}^{2} .
\end{array}\right.
$$

Donc $-2=p y_{1}^{2}-q y_{2}^{2}$. Ceci implique que $\left(\frac{p}{q}\right)=\left(\frac{2}{p}\right)=1$, ce qui n'est pas possible. Si $2 p(x-1)$ est un carré dans $\mathbb{N}$, on obtient la même contradiction.

(b) $q(x-1)$ ou $2 q(x-1)$ est un carré dans $\mathbb{N}$. Si $2 q(x-1)$ est un carré dans $\mathbb{N}$, alors il existe $\left(y_{1}, y_{2}\right) \in \mathbb{Z}^{2}$ tel que

$$
\left\{\begin{array}{l}
x-1=2 q y_{1}^{2} \\
x+1=2 p y_{2}^{2} .
\end{array}\right.
$$

Donc $1=p y_{2}^{2}-q y_{1}^{2}$. Ceci implique que $\left(\frac{p}{q}\right)=1$, ce qui n'est pas possible. Si $q(x-1)$ est un carré dans $\mathbb{N}$, on obtient la même contradiction.

Ainsi donc $p q(x-1)$ ou $(x-1)$ est un carré dans $\mathbb{N}$; ce qui est équivalent à dire que $2 \varepsilon_{3}$ est un carré dans $\mathbb{Q}(\sqrt{p q})$ (c.a.d. $\left.Q=2\right)$.

3. Nombre de classes capitulant dans certaines extensions qudratiques de $\mathbb{k}$. Soient $p$ un nombre premier congru à 1 modulo 4 et $q$ un nombre premier congru à -1 modulo $4, \mathbb{k}=\mathbb{Q}(\sqrt{2 p q}, i)$ tel que au moins deux éléments de $\left\{\left(\frac{2}{p}\right),\left(\frac{2}{q}\right),\left(\frac{p}{q}\right)\right\}$ valent -1 et l'indice $Q$ des unités de $\mathbb{Q}(\sqrt{2 p q})$ dans $\mathbb{k}$ est égal à 1 , et $\mathbb{k}_{2}^{(1)}$ le 2 -corps de classes de $\mathbb{k}$. D'après [Az-93], la 2-partie du groupe de classes $\mathbf{C}_{2}$ est isomorphe à $\mathbb{Z} / 2 \mathbb{Z} \times \mathbb{Z} / 2 \mathbb{Z}$. Dans notre cas $\mathbb{k}_{2}^{(1)}=\mathbb{Q}(\sqrt{p}, \sqrt{q}, \sqrt{2}, i)$ et les sous-extensions quadratiques de $\mathbb{k}_{2}^{(1)} / \mathbb{k}$ sont $: \mathbb{K}_{1}=\mathbb{Q}(\sqrt{p}, \sqrt{2 q}, i), \mathbb{K}_{2}=\mathbb{Q}(\sqrt{q}, \sqrt{2 p}, i)$ et $\mathbb{K}_{3}=\mathbb{Q}(\sqrt{2}, \sqrt{p q}, i)$.

On va faire une étude du problème de la capitulation dans les différentes sous-extensions quadratiques $\mathbb{K} / \mathbb{k}$ de $\mathbb{k}_{2}^{(1)} / \mathbb{k}$. 


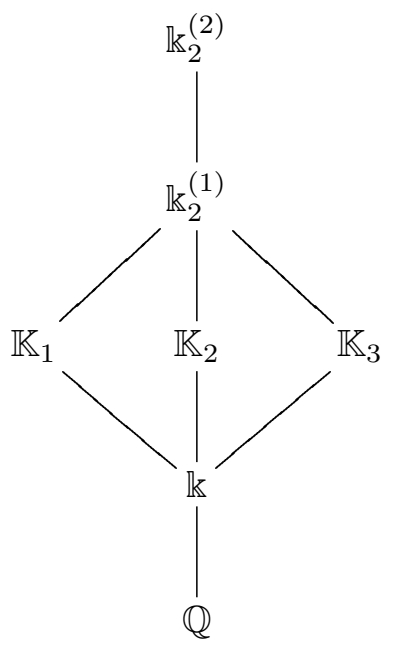

Diagramme 1

(a) Capitulation dans $\mathbb{K}_{1} / \mathbb{k}$. Soit $\varepsilon_{1}$ (resp. $\varepsilon_{2}, \varepsilon_{3}$ ) l'unité fondamentale de $\mathbb{Q}(\sqrt{p})$ (resp. $\mathbb{Q}(\sqrt{2 q}), \mathbb{Q}(\sqrt{2 p q}))$. On pose $\varepsilon_{3}=s+t \sqrt{2 p q}$. D'après le théorème 10, un $\mathrm{SFU}$ de $\mathbb{K}_{1}$ est donné de la façon suivante :

(i) Si $2 p(s \pm 1)$ est un carré dans $\mathbb{N}$, alors $\left\{\varepsilon_{1}, \sqrt{\varepsilon_{3}}, \sqrt{i \varepsilon_{2}}\right\}$ est un SFU de $\mathbb{K}_{1}$.

(ii) Si $2 q(s \pm 1)$ est un carré dans $\mathbb{N}$, alors $\left\{\varepsilon_{1}, \sqrt{i \varepsilon_{2}}, \sqrt{\varepsilon_{2} \varepsilon_{3}}\right\}$ est un SFU de $\mathbb{K}_{1}$.

Dans les deux cas on a $N\left(\mathbf{E}_{\mathbb{K}_{1}}\right)=\mathbf{E}_{\mathbb{k}}$. D'où, d'après le théorème 1, exactement deux classes de $\mathbf{C}_{2}$ capitulent dans $\mathbb{K}_{1}$.

(b) Capitulation dans $\mathbb{K}_{2} / \mathbb{k}$. Soit $\varepsilon_{1}$ (resp. $\varepsilon_{2}, \varepsilon_{3}$ ) l'unité fondamentale de $\mathbb{Q}(\sqrt{q})$ (resp. $\mathbb{Q}(\sqrt{2 p}), \mathbb{Q}(\sqrt{2 p q}))$. On pose $\varepsilon_{3}=s+t \sqrt{2 p q}$. Distinguons les deux cas suivants:

(i) On suppose que $\varepsilon_{2}$ est de norme -1 (théorème 11). Dans ce cas :

- si $2 q(s \pm 1)$ est un carré dans $\mathbb{N}$, alors $\left\{\sqrt{i \varepsilon_{1}}, \varepsilon_{2}, \sqrt{\varepsilon_{3}}\right\}$ est un SFU de $\mathbb{K}_{2}$;

- si $2 p(s \pm 1)$ est un carré dans $\mathbb{N}$, alors $\left\{\sqrt{i \varepsilon_{1}}, \varepsilon_{2}, \sqrt{\varepsilon_{1} \varepsilon_{3}}\right\}$ est un SFU de $\mathbb{K}_{2}$.

(ii) On suppose que $\varepsilon_{2}$ est de norme 1 (lemme 8, théorème 9). Dans ce cas :

- si $2 p(s \pm 1)$ est un carré dans $\mathbb{N}$, alors $\left\{\sqrt{i \varepsilon_{1}}, \sqrt{i \varepsilon_{2}}, \sqrt{i \varepsilon_{3}}\right\}$ est un SFU de $\mathbb{K}_{2}$;

- si $2 q(s \pm 1)$ est un carré dans $\mathbb{N}$, alors $\left\{\sqrt{i \varepsilon_{1}}, \sqrt{i \varepsilon_{2}}, \sqrt{\varepsilon_{3}}\right\}$ est un SFU de $\mathbb{K}_{2}$. 
Dans tous ces cas on a que $N\left(\mathbf{E}_{\mathbb{K}_{2}}\right)=\mathbf{E}_{\mathbb{k}}$. Par suite, d'après le théorème 1, exactement deux classes de $\mathbf{C}_{2}$ capitulent dans $\mathbb{K}_{2}$.

(c) Capitulation dans $\mathbb{K}_{3}$. Soit $\varepsilon_{1}$ (resp. $\varepsilon_{2}, \varepsilon_{3}$ ) l'unité fondamentale de $\mathbb{Q}(\sqrt{2})$ (resp. $\mathbb{Q}(\sqrt{p q}), \mathbb{Q}(\sqrt{2 p q})$ ). On pose $\varepsilon_{2}=x+y \sqrt{p q}$. D'après le théorème 12 , un $\mathrm{SFU}$ de $\mathbb{K}_{3}$ est donné de la façon suivante :

(i) Si $x \pm 1$ est un carré dans $\mathbb{N}$, alors $\left\{\varepsilon_{1}, \sqrt{\varepsilon_{2}}, \varepsilon_{3}\right\}$ est un SFU de $\mathbb{K}_{3}$.

(ii) Sinon, $\left\{\varepsilon_{1}, \varepsilon_{2}, \sqrt{\varepsilon_{2} \varepsilon_{3}}\right\}$ est un SFU de $\mathbb{K}_{3}$.

Donc, si $x \pm 1$ est un carré dans $\mathbb{N}$, alors $\left[\mathbf{E}_{\mathbb{k}}: N\left(\mathbf{E}_{\mathbb{K}_{3}}\right)\right]=2 ;$ sinon, $N\left(\mathbf{E}_{\mathbb{K}_{3}}\right)=$ $\mathbf{E}_{\mathbb{k}}$. Il vient, d'après le théorème 1 , que toutes les classes de $\mathbf{C}_{2}$ capitulent dans $\mathbb{K}_{3}$ si et seulement si $x \pm 1$ est un carré dans $\mathbb{N}$.

On obtient alors le théorème suivant :

ThÉORÈme 16 . Soient $\mathbb{k}=\mathbb{Q}(\sqrt{2 p q}, i)$ avec au moins deux éléments de $\left\{\left(\frac{2}{p}\right),\left(\frac{2}{q}\right),\left(\frac{p}{q}\right)\right\}$ valant -1 et l'indice $Q$ des unités de $\mathbb{Q}(\sqrt{2 p q})$ dans $\mathbb{k}$ est égal à $1, \mathbb{K}_{1}=\mathbb{Q}(\sqrt{p}, \sqrt{2 q}, i), \mathbb{K}_{2}=\mathbb{Q}(\sqrt{q}, \sqrt{2 p}, i)$ et $\mathbb{K}_{3}=\mathbb{Q}(\sqrt{2}, \sqrt{p q}, i)$. Alors dans chaque extension $\mathbb{K}_{i}, i \in\{1,2,3\}$, il existe exactement deux classes de $\mathbf{C}_{2}$ qui capitulent.

Preuve. Soit $\varepsilon_{2}=x+y \sqrt{p q}$ (resp. $\varepsilon_{3}=s+t \sqrt{2 p q}$ ) l'unité fondamentale de $\mathbb{Q}(\sqrt{p q})($ resp. $\mathbb{Q}(\sqrt{2 p q}))$. On rappelle que $p \equiv-q \equiv 1 \bmod 4$. On désigne par $\left(\frac{m}{p}\right)$ le symbole de Legendre pour un entier naturel $m$. On sait que deux classes seulement capitulent dans $\mathbb{K}_{1}$, que deux classes seulement capitulent dans $\mathbb{K}_{2}$ et que les 4 classes de $\mathbf{C}_{2}$ capitulent dans $\mathbb{K}_{3}$ si et seulement si $x \pm 1$ est un carré dans $\mathbb{N}$. On se propose de montrer que $x \pm 1$ n'est jamais un carré de $\mathbb{N}$.

Montrons que si $x \pm 1$ est un carré dans $\mathbb{N}$, alors $\left(\frac{2}{p}\right)=1$. En effet, $(x-1)(x+1)=p q y^{2}$. Si $x \pm 1$ est un carré dans $\mathbb{N}$, alors il existe $\left(y_{1}, y_{2}\right) \in \mathbb{N}^{2}$ tel que l'une des deux situations suivantes est satisfaite :

$$
\begin{aligned}
& \left\{\begin{array}{l}
x-1=p q y_{1}^{2}, \\
x+1=y_{2}^{2},
\end{array} \quad 1=\left(\frac{y_{2}^{2}}{p}\right)=\left(\frac{x+1}{p}\right)=\left(\frac{x-1+2}{p}\right)=\left(\frac{2}{p}\right) ;\right. \\
& \left\{\begin{array}{l}
x+1=p q y_{1}^{2}, \\
x-1=y_{2}^{2},
\end{array} \quad 1=\left(\frac{y_{2}^{2}}{p}\right)=\left(\frac{x-1}{p}\right)=\left(\frac{x+1-2}{p}\right)=\left(\frac{-2}{p}\right)=\left(\frac{2}{p}\right) .\right.
\end{aligned}
$$

Ainsi, cette propriété est démontrée.

Montrons que si $2 \varepsilon_{3}$ n'est pas un carré dans $\mathbb{Q}(\sqrt{2 p q})$, alors $\left(\frac{q}{p}\right)=1$ ou bien $\left(\frac{2}{p}\right)=\left(\frac{q}{p}\right)$. En effet, $(s-1)(s+1)=2 p q t^{2}$. Si de plus $2 \varepsilon_{3}$ n'est pas un carré dans $\mathbb{Q}(\sqrt{2 p q})$, alors $s \pm 1$ n'est pas un carré dans $\mathbb{N}$ et il existe $\left(t_{1}, t_{2}\right) \in \mathbb{N}^{2}$ tel qu'on a l'une des situations suivantes: 


$$
\begin{aligned}
& \left\{\begin{array}{l}
s \pm 1=2 p t_{1}^{2}, \\
s \mp 1=q t_{2}^{2},
\end{array} \quad\left(\frac{q}{p}\right)=\left(\frac{s \mp 1}{p}\right)=\left(\frac{s \pm 1 \mp 2}{p}\right)=\left(\frac{2}{p}\right) .\right. \\
& \left\{\begin{array}{l}
s \pm 1=p t_{1}^{2}, \\
s \mp 1=2 q t_{2}^{2},
\end{array} \quad\left(\frac{2 q}{p}\right)=\left(\frac{s \mp 1}{p}\right)=\left(\frac{s \pm 1 \mp 2}{p}\right)=\left(\frac{2}{p}\right) \Rightarrow\left(\frac{q}{p}\right)=1 .\right.
\end{aligned}
$$

Ceci termine la démonstration de cette propriété.

Conclusion : Si $x \pm 1$ est un carré dans $\mathbb{N}$, alors, d'après les deux propriétés précédentes, $\left(\frac{q}{p}\right)=\left(\frac{2}{p}\right)=1$. Par conséquent, nos hypothèses ne seront pas vérifiées. Il en résulte que $x \pm 1$ ne peut pas être un carré dans $\mathbb{N}$.

4. Structure de $G_{2}$ et 2-groupe de classes de $\mathbb{k}$. Soient $p$ un nombre premier congru à 1 modulo 4 et $q$ un nombre premier congru à -1 modulo $4, \mathbb{k}=\mathbb{Q}(\sqrt{2 p q}, i)$ tel que au moins deux éléments de $\left\{\left(\frac{2}{p}\right),\left(\frac{2}{q}\right),\left(\frac{p}{q}\right)\right\}$ valent -1 et l'indice $Q$ des unités de $\mathbb{Q}(\sqrt{2 p q})$ dans $\mathbb{k}$ est égal à $1, \mathbf{C}_{2}$ le 2 -groupe des classes de $\mathbb{k}, \mathbf{E}_{\mathbb{k}}$ le groupe des unités de $\mathbb{k}, \mathbb{k}_{2}^{(1)}$ le 2-corps de classes de Hilbert de $\mathbb{k}$ et $G_{2}$ le groupe de Galois de $\mathbb{k}_{2}^{(2)} / \mathbb{k}$.

Nous étudions la capitulation de certains idéaux de $\mathbb{k}$ et par la suite nous déterminons la structure de $G_{2}$ en nous basant sur le théorème 2 .

Proposition 17. Soient $\mathbb{k}=\mathbb{Q}(\sqrt{2 p q}, i)$ et $\mathcal{H}_{0}$ l'idéal premier au dessus de $1+i$ dans $\mathbb{k}$. Alors la classe de $\mathcal{H}_{0}$ dans $\mathbb{k}$ est d'ordre 2 . De plus $\mathcal{H}_{0}$ capitule dans $\mathbb{k}(\sqrt{2})$.

Preuve. Montrons que la classe de $\mathcal{H}_{0}$ est d'ordre 2. Comme 2 est totalement ramifié dans $\mathbb{k} / \mathbb{Q}$, alors il existe un idéal $\mathcal{H}_{0}$ de $\mathbb{k}$ tel que $\mathcal{H}_{0}^{2}=$ $(1+i)$. On suppose que $\mathcal{H}_{0}=(\alpha)$ pour un certain $\alpha$ dans $\mathbb{k}$, ce qui est équivalent à $\left(\alpha^{2}\right)=(1+i)$. Il existe une unité $\varepsilon$ de $\mathbb{k}$ telle que $(1+i) \varepsilon=\alpha^{2}$. Comme l'indice des unités $Q$ est égal à 1 , alors $\varepsilon$ est un nombre réel ou bien un nombre purement imaginaire. Les deux cas se traitent de la même façon. Supposons que $\varepsilon$ est réel. Soit $\alpha=\alpha_{1}+i \alpha_{2}$ avec $\alpha_{1}$ et $\alpha_{2}$ deux nombres réels de $\mathbb{k}$. On a

$$
\left\{\begin{array}{l}
\varepsilon=\alpha_{1}^{2}-\alpha_{2}^{2}, \quad \alpha_{1}^{2}-\alpha_{2}^{2}-2 \alpha_{1} \alpha_{2}=0 \text { et } \alpha_{1}-\alpha_{2}= \pm \alpha_{2} \sqrt{2} . \\
\varepsilon=2 \alpha_{1} \alpha_{2},
\end{array}\right.
$$

Comme $\sqrt{2} \notin \mathbb{k}$, alors on a une contradiction. Il s'ensuit que la classe de $\mathcal{H}_{0}$ est d'ordre 2 .

Montrons que $\mathcal{H}_{0}$ capitule dans $\mathbb{k}(\sqrt{2})$. Comme dans le cas précédent, le problème est de chercher un élément $\alpha$ de $\mathbb{k}(\sqrt{2})$ tel que $\left(\alpha^{2}\right)=(1+i)$. Ceci est équivalent à $(1+i) \varepsilon=\alpha^{2}$ pour une certaine unité $\varepsilon$ de $\mathbb{k}(\sqrt{2})$. Si $\varepsilon$ est réel et $\alpha=\alpha_{1}+i \alpha_{2}$, on trouve que

$$
\left\{\begin{array} { l } 
{ \varepsilon = \alpha _ { 1 } ^ { 2 } - \alpha _ { 2 } ^ { 2 } , } \\
{ \varepsilon = 2 \alpha _ { 1 } \alpha _ { 2 } , }
\end{array} \quad \text { ce qui est équivalent à } \quad \left\{\begin{array}{l}
\alpha_{1}=\sqrt{\varepsilon(1 \pm \sqrt{2}) / 2,} \\
\alpha_{2}=\varepsilon /\left(2 \alpha_{1}\right) .
\end{array}\right.\right.
$$


Il est clair que si $\varepsilon=1 \pm \sqrt{2}$, alors $\alpha_{1}$ et $\alpha_{2}$ appartiennent à $\mathbb{k}(\sqrt{2})$ et par conséquent $\left(\mathcal{H}_{0}\right)=(\alpha)$ dans $\mathbb{k}(\sqrt{2})$. D'où le résultat.

Proposition 18. Soient $\mathbb{k}=\mathbb{Q}(\sqrt{2 p q}, i)$, $b$ et $c$ deux entiers tels que $p=b^{2}+c^{2}$ et $\mathcal{P}$ l'idéal premier de $\mathbb{k}$ au-dessus de $b+c i$. Alors la classe de $\mathcal{P}$ est d'ordre 2 dans $\mathbb{k}$. De plus, l'idéal $\mathcal{P}$ devient principal dans $\mathbb{k}(\sqrt{p})$.

Preuve. Comme $p$ est ramifié dans $\mathbb{k} / \mathbb{Q}(i)$, alors il existe un idéal $\mathcal{P}$, premier dans $\mathbb{k}$ et tel que $(b+c i)=\mathcal{P}^{2}$. Si $\mathcal{P}$ est principal dans $\mathbb{k}$, alors il existe $\alpha$ dans $\mathbb{k}$ et $\varepsilon$ une unité de $\mathbb{k}$ tels que $(b+c i) \varepsilon=\alpha^{2}$. Comme l'indice des unités $Q$ est égal à 1 , alors $\varepsilon$ est une unité réelle ou bien une unité purement imaginaire. Les deux cas se traitent de la même façon. On suppose que $\varepsilon$ est une unité réelle. Si $\alpha=\alpha_{1}+i \alpha_{2}$ avec $\alpha_{1}$ et $\alpha_{2}$ deux nombres réels de $\mathbb{k}$, alors

$$
\left\{\begin{array}{l}
b \varepsilon=\alpha_{1}^{2}-\alpha_{2}^{2}, \quad c \alpha_{1}^{2}-c \alpha_{2}^{2}-2 b \alpha_{1} \alpha_{2}=0 \text { et } \alpha_{1}=\frac{2 \alpha_{2}(b \pm \sqrt{p})}{2 c} . \\
c \varepsilon=2 \alpha_{1} \alpha_{2},
\end{array} .\right.
$$

Or ceci implique que $\sqrt{p} \in \mathbb{k}$. Donc on a une contradiction. Par suite la classe de $\mathcal{P}$ est d'ordre 2. Montrons que l'idéal $\mathcal{P}$ devient principal dans $\mathbb{k}(\sqrt{p})$.

Soit $\varepsilon_{1}$ l'unité fondamentale de $\mathbb{Q}(\sqrt{p})$. Deux situations se présentent.

1) Cas où $\varepsilon_{1}=x+y \sqrt{p}$ avec $x$ et $y$ dans $\mathbb{Z}$. Dans ce cas $(x-i)(x+i)=p y^{2}$ et le plus grand commun diviseur de $x-i$ et $x+i$ est un diviseur de 2. Par suite,

$$
\left\{\begin{array}{l}
x-i=\pi y_{1}^{2} \\
x+i=\pi^{\prime} y_{2}^{2}
\end{array}\right.
$$

avec $\pi=b+c i$ ou $i(b+c i)$, où $\pi^{\prime}$ est le conjugué de $\pi$ et $y=y_{1} y_{2}$.

$\mathrm{Vu}$ que $c$ et $b$ jouent un rôle symétrique, on suppose que $\pi=b+c i$. Dans ces conditions, $\sqrt{\varepsilon_{1}}=\frac{1}{2}\left(y_{1} \sqrt{2 \pi}+y_{2} \sqrt{2 \pi^{\prime}}\right)$. D'autre part, $\mathcal{P}$ devient principal dans $\mathbb{k}(\sqrt{p})$ si et seulement s'il existe $\alpha$ dans $\mathbb{k}(\sqrt{p})$ et $\varepsilon^{\prime}$ une unité de $\mathbb{k}(\sqrt{p})$ tels que $\pi \varepsilon^{\prime}=\alpha^{2}$. Si $\alpha=\alpha_{1}+\alpha_{2} i$ et $\varepsilon^{\prime}=\varepsilon i$ avec $\varepsilon$ une unité réelle, alors on trouve que $\alpha_{1}=\sqrt{\varepsilon(-c \pm \sqrt{p}) / 2}$ et $\alpha_{2}=b \varepsilon /\left(2 \alpha_{1}\right)$. De plus, $\sqrt{-c+\sqrt{p}}=\frac{1}{1+i}\left(\sqrt{-\pi}+\sqrt{\pi^{\prime}}\right)$. Si on prend $\varepsilon=\varepsilon_{1}$, alors $\alpha_{1}$ et $\alpha_{2}$ appartiennent à $\mathbb{k}(\sqrt{p})$. D'où l'idéal engendré par $\mathcal{P}$ dans $\mathbb{k}(\sqrt{p})$ est principal.

2) Cas où $\varepsilon_{1}=\frac{1}{2}(x+y \sqrt{p})$. De la même façon que précédemment, on trouve que

$$
\left\{\begin{array}{l}
x-2 i=\pi y_{1}^{2} \\
x+2 i=\pi^{\prime} y_{2}^{2}
\end{array}\right.
$$

avec $\pi=b+c i$ ou $i(b+c i)$, où $\pi^{\prime}$ est le conjugué de $\pi$ et $y=y_{1} y_{2}$.

On suppose que $\pi=b+c i$. Alors $\sqrt{\varepsilon_{1}}=\frac{1}{2}\left(y_{1} \sqrt{\pi}+y_{2} \sqrt{\pi^{\prime}}\right)$. De même, $\mathcal{P}$ devient principal dans $\mathbb{k}(\sqrt{p})$ si et seulement si l'équation en $\alpha, \pi \varepsilon=\alpha^{2}$ où 
$\varepsilon$ est une unité de $\mathbb{k}(\sqrt{p})$, est résoluble dans $\mathbb{k}(\sqrt{p})$. Si $\alpha=\alpha_{1}+\alpha_{2} i$ et $\varepsilon$ est une unité réelle, alors on trouve que $\alpha_{1}=\sqrt{\varepsilon(b \pm \sqrt{p}) / 2}$ et $\alpha_{2}=c \varepsilon /\left(2 \alpha_{1}\right)$. Comme $\sqrt{b+\sqrt{p}}=\sqrt{\pi / 2}+\sqrt{\pi^{\prime} / 2}$ et pour $\varepsilon=\varepsilon_{1}$, les nombres $\alpha_{1}$ et $\alpha_{2}$ appartiennent à $\mathbb{k}(\sqrt{p})$, alors $\mathcal{P}$ devient principal dans $\mathbb{k}(\sqrt{p})$.

Proposition 19. Soient $\mathbb{k}=\mathbb{Q}(\sqrt{2 p q}, i), \mathcal{H}_{0}$ l'idéal premier de $\mathbb{k}$ audessus de $1+i$ et $\mathcal{P}$ l'idéal premier de $\mathbb{k}$ au-dessus de $b+c i$ où $b$ et $c$ sont deux entiers tels que $b^{2}+c^{2}=p$. Alors la classe de $\mathcal{H}_{0} \mathcal{P}$ est d'ordre 2 dans $\mathbb{k}$ et la 2-partie du groupe des classes de $\mathbb{k}$ est engendrée par les classes de $\mathcal{H}_{0}$ et $\mathcal{P}$.

Preuve. On pose $b^{\prime}=b-c$ et $c^{\prime}=b+c$. Alors $b^{\prime 2}+c^{\prime 2}=2 p$. L'idéal $\mathcal{H}_{0} \mathcal{P}=(\alpha)$ où $\alpha$ appartient à $\mathbb{k}$ si et seulement s'il existe une unité $\varepsilon$ dans $\mathbb{k}$ telle que $(1+i)(b+c i) \varepsilon=\alpha^{2}$. Comme l'indice des unités $Q$ est égal à 1 , alors $\varepsilon$ est une unité réelle ou bien une unité purement imaginaire. Les deux cas se traitent de la même façon. Par suite, on suppose que $\varepsilon$ est une unité réelle. Si $\alpha=\alpha_{1}+i \alpha_{2}$ avec $\alpha_{1}$ et $\alpha_{2}$ deux nombres réels de $\mathbb{k}$, alors

$$
\left\{\begin{array}{l}
b^{\prime} \varepsilon=\alpha_{1}^{2}-\alpha_{2}^{2}, \quad c^{\prime} \alpha_{1}^{2}-c^{\prime} \alpha_{2}^{2}-2 b^{\prime} \alpha_{1} \alpha_{2}=0, \\
c^{\prime} \varepsilon=2 \alpha_{1} \alpha_{2},
\end{array}\right.
$$

donc $\alpha_{1}=\alpha_{2}\left(b^{\prime} \pm \sqrt{2 p}\right) / c^{\prime}$. Or ceci implique que $\sqrt{2 p} \in \mathbb{k}$. Donc on a une contradiction. Par conséquent, la classe de $\mathcal{H}_{0} \mathcal{P}$ est d'ordre deux dans $\mathbb{k}$ et la 2-partie du groupe des classes de $\mathbb{k}$ est engendrée par les classes de $\mathcal{H}_{0}$ et de $\mathcal{P}$.

Proposition 20. On garde les notations et les hypothèses des propositions précédentes et on suppose de plus que $\left(\frac{2}{p}\right)=-1$. Alors l'idéal $\mathcal{H}_{0} \mathcal{P}$ capitule dans $\mathbb{k}(\sqrt{2 p})$.

Preuve. Dans ce cas l'unité fondamentale de $\mathbb{Q}(\sqrt{2 p}), \varepsilon_{2}=x+y \sqrt{2 p}$, est de norme -1 . D'où

$$
\left\{\begin{array}{l}
x-i=(1+i) \pi y_{1}^{2}, \\
x+i=(1-i) \pi^{\prime} y_{2}^{2},
\end{array}\right.
$$

avec $\pi=b+c i$ ou $i(b+c i)$, où $\pi^{\prime}$ est le conjugué de $\pi$ et $y=y_{1} y_{2}$. Comme $b$ et $c$ jouent un rôle symétrique, on suppose que $\pi=b+c i$. Alors on a que $\sqrt{\varepsilon_{2}}=\frac{1}{2}\left(y_{1} \sqrt{(1+i) \pi}+y_{2} \sqrt{(1-i) \pi^{\prime}}\right)$. D'autre part, l'idéal $\mathcal{H}_{0} \mathcal{P}$ devient principal dans $\mathbb{k}(\sqrt{2 p})$ si et seulement si l'équation en $\alpha,(1+i) \pi \varepsilon=\alpha^{2}$ où $\varepsilon$ est une unité de $\mathbb{k}(\sqrt{2 p})$, est résoluble dans $\mathbb{k}(\sqrt{2 p})$. Si $\alpha=\alpha_{1}+\alpha_{2} i$ avec $\alpha_{1}$ et $\alpha_{2}$ deux nombres réels et $\varepsilon$ est une unité réelle, alors

$$
\alpha_{1}=\sqrt{\frac{\varepsilon\left(b^{\prime} \pm \sqrt{2 p}\right)}{2}} \quad \text { et } \quad \alpha_{2}=\frac{c^{\prime} \varepsilon}{2 \alpha_{1}} \quad \text { avec } b^{\prime}=b-c \text { et } c^{\prime}=b+c .
$$


De plus,

$$
\sqrt{b^{\prime}+\sqrt{2 p}}=\sqrt{\frac{(1+i) \pi}{2}}+\sqrt{\frac{(1-i) \pi^{\prime}}{2}} .
$$

Il est clair que si $\varepsilon=\varepsilon_{2}$, alors $\alpha_{1}$ et $\alpha_{2}$ appartiennent à $\mathbb{k}(\sqrt{2 p})$. Par suite, l'idéal $\mathcal{H}_{0} \mathcal{P}$ devient principal dans $\mathbb{k}(\sqrt{2 p})$.

ThÉORÈme 21. Soient $\mathbb{k}=\mathbb{Q}(\sqrt{2 p q}, i), \mathbb{k}_{2}^{(1)}$ le 2 -corps de classes de Hilbert de $\mathbb{k}, \mathbb{k}_{2}^{(2)}$ le 2-corps des classes de Hilbert de $\mathbb{k}_{2}^{(1)}$ et $G_{2}$ le groupe de Galois de $\mathbb{k}_{2}^{(2)} / \mathbb{k}$. Suivant trois cas, le type des sous-corps de $\mathbb{k}_{2}^{(1)}$, quadratique sur $\mathbb{k}$, est donné dans le tableau ci-dessous:

\begin{tabular}{lccc}
\hline & $\mathbb{k}(\sqrt{2})$ & $\mathbb{k}(\sqrt{p})$ & $\mathbb{k}(\sqrt{2 p})$ \\
\hline$\left(\frac{2}{p}\right)=\left(\frac{2}{q}\right)=\left(\frac{p}{q}\right)=-1$ & $A$ & $A$ & $A$ \\
\hline$\left(\frac{2}{p}\right)=\left(\frac{2}{q}\right)=-1,\left(\frac{p}{q}\right)=1$ & $A$ & $B$ & $B$ \\
\hline$\left(\frac{2}{p}\right)=\left(\frac{p}{q}\right)=-1,\left(\frac{2}{q}\right)=1$ & $B$ & $A$ & $B$ \\
\hline
\end{tabular}

En particulier, si $\mathbb{F}=\mathbb{k}(\sqrt{2 p})$ et $\mathbf{C}_{\mathbb{k}, \mathbb{F}}$ est le sous-groupe de $\mathbf{C}_{\mathbb{k}}$ associé $\grave{a} \mathbb{F}$ par la théorie des corps de classes, alors dans le premier cas, $\mathbf{C}_{\mathbb{k}, \mathbb{F}}$ ne contient ni $\mathcal{H}_{0}$ ni $\mathcal{P}$, tandis que dans les deux autres cas il contient exactement l'un d'eux. De plus, le groupe $G_{2}$ est isomorphe au groupe des quaternions $Q_{3}$ dans le premier cas, tandis que dans les deux autres cas il est isomorphe à $Q_{m}$ où $4 \leq m$.

Preuve. Soient $b$ et $c$ deux entiers tels que $p=b^{2}+c^{2}, \mathcal{H}_{0}$ l'idéal premier de $\mathbb{k}$ au-dessus de $(1+i)$ et $\mathcal{P}$ l'idéal premier de $\mathbb{k}$ au-dessus de $(b+c i)$. On remarque les faits suivants.

L'idéal $\mathcal{H}_{0}$ capitule dans $\mathbb{k}(\sqrt{2})$ et il se décompose complètement dans $\mathbb{k}(\sqrt{2}) / \mathbb{k}$ si et seulement $\left(\frac{2}{p}\right)=\left(\frac{2}{q}\right)=-1$. Dans ce cas, le corps $\mathbb{k}(\sqrt{2})$ est de type (A). Dans le cas contraire, $\mathbb{k}(\sqrt{2})$ est de type (B).

De plus, si $\left(\frac{2 q}{p}\right)=1$, alors $\mathcal{P}$ capitule dans $\mathbb{k}(\sqrt{p})=\mathbb{Q}(\sqrt{p}, \sqrt{2 q}, i)$ et se décompose complètement dans $\mathbb{k}(\sqrt{p}) / \mathbb{k}$. D'où $\mathcal{P}$ est norme dans $\mathbb{k}(\sqrt{p}) / \mathbb{k}$. Donc le corps $\mathbb{k}(\sqrt{p})$ est de type (A). Dans le cas contraire, $\mathbb{k}(\sqrt{p})$ est de type (B).

Si $\left(\frac{2 q}{p}\right)=-1$, on a :

1) Soit $p \equiv 1 \bmod 8$ et $\left(\frac{q}{p}\right)=-1$. Alors, d'après la preuve du théorème 16 , la condition $Q=1$ entraîne que $\left(\frac{q}{p}\right)=1$ ou bien $\left(\frac{2}{p}\right)=\left(\frac{q}{p}\right)$. Par suite ce cas est impossible. 
2) Soit $p \equiv 5 \bmod 8$ et $\left(\frac{q}{p}\right)=1$. Alors $\mathcal{H}_{0} \mathcal{P}$ capitule dans $\mathbb{k}(\sqrt{2 p})$. Comme $\mathcal{H}_{0}$ est inerte dans $\mathbb{k}(\sqrt{2 p}) / \mathbb{k}$, alors $\mathcal{H}_{0} \mathcal{P}$ n'est pas norme dans $\mathbb{k}(\sqrt{2 p}) / \mathbb{k}$. Par suite, $\mathbb{k}(\sqrt{2 p})$ est de type (B). De plus, dans ce cas $\mathbb{k}(\sqrt{2})$ est de type (A).

En utilisant le théorème 4 le résultat s'ensuit.

Remerciement. Je remercie vivement le rapporteur de mon article pour ses precieuses remarques.

\section{Bibliographie}

[Az-93] A. Azizi, Capitulation des 2-classes d'idéaux de $\mathbb{Q}(\sqrt{d}, i)$, thèse, Université Laval-Québec-Canada, 1993.

[Az-97] —, Sur la capitulation des 2-classes d'idéaux de $\mathbb{Q}(\sqrt{d}, i)$, C. R. Acad. Sci. Paris Sér. I 325 (1997), 127-130.

[Az-99:1] - Sur le groupe de classes d'idéaux de $\mathbb{Q}(\sqrt{d}, i)$, Rend. Circ. Mat. Palermo (2) 48 (1999), 71-92.

[Az-99:2] -, Unités de certains corps de nombres imaginaires et abéliens sur $\mathbb{Q}$, Ann. Sci. Math. Québec 23 (1999), 87-93.

[H-Y-90] M. Hirabayashi and K. I. Yoshino, Unit indices of imaginary abelian number fields of type $(2,2,2)$, J. Number Theory 34 (1990), 346-361.

[Ki-76] H. Kisilevsky, Number fields with class number congruent to 4 mod 8 and Hilbert's theorem 94, ibid. 8 (1976), 271-279.

[Kub-56] T. Kubota, Über den bizyklischen biquadratischen Zahlkörper, Nagoya Math. J. 10 (1956), 65-85.

[Kur-43] S. Kuroda, Über den Dirichletschen Körper, J. Fac. Sci. Imp. Univ. Tokyo Sec. I 4 (1943), 383-406.

[Mi-89] K. Miyake, Algebraic investigations of Hilbert's theorem 94, the principal ideal theorem and capitulation problem, Exposition. Math. 7 (1989), 289-346.

[Su-91] H. Suzuki, A generalisation of Hilbert's theorem 94, Nagoya Math. J. 121 (1991), 161-169.

[Te-71] F. Terada, A principal ideal theorem in the genus fields, Tôhoku Math. J. (2) 23 (1971), 697-718.

Département de Mathématiques

Faculté des Sciences

Université Mohammed 1

Oujda, Maroc

E-mail: azizi@sciences.univ-oujda.ac.ma 